\title{
Isochlorogenic Acid C Reverses Epithelial-Mesenchymal Transition via Down-regulation of EGFR Pathway in MDA-MB-231 cells
}

\author{
JI-KUEN YU ${ }^{1}$, CHIA-HERNG YUE ${ }^{1}$, YING-RU PAN ${ }^{2}$, YUNG-WEI CHIU ${ }^{3}$, \\ JER-YUH LIU ${ }^{4,5}$, KUN-I LIN ${ }^{6,7}$ and CHIA-JEN LEE ${ }^{2}$ \\ ${ }^{1}$ Department of Surgery, Tungs' Taichung MetroHarbor Hospital, Taichung, Taiwan, R.O.C; \\ ${ }^{2}$ Department of Medical Research, Tung's Taichung MetroHarbor Hospital, Taichung, Taiwan, R.O.C.; \\ ${ }^{3}$ Emergency Department and Center of Hyperbaric Oxygen Therapy, \\ Tungs' Taichung Metro Harbor Hospital, Taichung, Taiwan, R.O.C.; \\ ${ }^{4}$ Center for Molecular Medicine, China Medical University Hospital, Taichung, Taiwan, R.O.C.; \\ ${ }^{5}$ Graduate Institute of Biomedical Sciences, China Medical University, Taichung, Taiwan, R.O.C.; \\ ${ }^{6}$ Department of Obstetrics and Gynecology, \\ Chang Bing Show Chwan Memorial Hospital, Changhua, Taiwan, R.O.C.; \\ ${ }^{7}$ Department of Cosmetic Science, Providence University, Taichung, Taiwan, R.O.C.
}

\begin{abstract}
Background/Aim: Epidermal growth factor receptor (EGFR) has been suggested to play an important role in survival, proliferation, migration, differentiation, and tumorigenesis of many cell types. Breast cancer patients with high EGFR expression have a poor prognosis. In this study, we investigated the molecular mechanism of the inhibitory effect of isochlorogenic acid c (ICAC) extracted from Lonicera japonica on elevated EGFR levels of the triple-negative breast cancer (TNBC) cell line, MDA-MB-231. Materials and Methods: The cell viability and cell-cycle analysis were evaluated using 3-(4, 5-dimethyl-2-thiazolyl)-2, 5-diphenyl-2Htetrazolium bromide (MTT) assay and flow cytometry, respectively. The migration ability and invasiveness of ICACtreated MDA-MB-231 were examined by migration and Matrigel invasion assay. The epithelial-mesenchymal-transition (EMT)-related protein expression was examined by western blotting and reverse transcriptase-polymerase chain reaction
\end{abstract}

Correspondence to: Dr. Chia-Jen Lee, Department of Medical Research, Tungs' Taichung MetroHarbor Hospital, No.699, Sec. 8, Taiwan Blvd., Taichung City 435, Taiwan. Tel: +886 426581919 ext.79004, e-mail: chiajenlee54@gmail.com and Dr. Kun-I Lin, Department of Obstetrics and Gynecology, Chang Bing Show Chwan Memorial Hospital, No. 6 Lugong Road, Lugang Town, Changhua County, 505 Taiwan. Tel: +886 47812012, e-mail: kunidg107@gmail.com

Key Words: Isochlorogenic acid c (ICAC), epithelial-mesenchymal transition (EMT), epidermal growth factor receptor (EGFR), triplenegative breast cancer (TNBC), MDA-MB-231 cells.
(RT-PCR). Results: ICAC led to significant morphological changes and suppressed migration and invasion capacities of highly metastatic MDA-MB-231 cells. Western blot analysis for EGFR/EMT-associated proteins suggested that ICAC attenuated the mesenchymal traits as observed by upregulation of epithelial markers and down-regulation of mesenchymal markers as well as decreased activities of matrix metalloproteinase-9 (MMP-9). Conclusion: These results suggested that the inhibitory effects of ICAC against EGFRinduced EMT and MDA-MB-231 cell invasion were dependent on the EGFR/ phospholipase C $\gamma(P L C \gamma) /$ extracellular regulated protein kinase $1 / 2\left(E R K^{1 / 2}\right) /$ slug signaling pathway. Therefore, the obtained results could provide us clues for the next therapeutic strategy in the treatment of TNBC.

Triple-negative breast cancer (TNBC) is classified as a poorly differentiated and aggressive type of tumor. Approximately $12 \%$ to $17 \%$ of women with breast cancer belong to TNBC (1). TNBC is characterized by tumors that can not express estrogen receptor (ER), progesterone receptor $(\mathrm{PR})$ and lack human epidermal growth factor receptor type 2 (HER2, also known as ErbB2 or Neu) overexpression. Therefore, treating TNBC patients is pretty difficult due to the absence of a suitable target. Therapy for TNBC is limited to conventional chemotherapy; however, relapse leading to poor clinical outcome occurs frequently because of high rates of metastasis and general inaccuracy of chemotherapy $(2,3)$. This may be due to its high level of molecular heterogeneity that is the main reason why TNBC cannot be effectively treated. 
Epidermal growth factor receptor (EGFR) is a transmembrane tyrosine kinase that belongs to the HER/ErbB protein family. EGFR could be expressed in multiple types of cancer cells, including breast and lung cancers, which correlates with tumor development, progression and prognosis (4-6). The patients with EGFRmutated lung adenocarcinoma seemed to have a longer overall survival (OS) than those with wild-type EGFR (6). $E G F R$ mutation testing is considered to be helpful for increasing the number of patients with lung adenocarcinoma eligible for targeted therapy (7). Patients harboring EGFR mutations seemed to benefit more from the association of treatment outcomes in non-small cell lung cancer (NSCLC) with metastasis (8). EGFR and its downstream signaling pathways promote many cellular events considered hallmarks of cancerous behaviors in cells; including cell proliferation, survival, epithelial-to-mesenchymal transition (EMT), migration, invasion, tumor progression and drug resistance (9). TNBC cells are frequently expressed with high levels of EGFR and constitutive activation of MAPK signaling pathways (10-12). EGFR is one of the potential therapeutic targets for TNBC. However, EGFR-targeted therapies, including small molecular tyrosine kinase inhibitor (TKI) and monoclonal antibody (mAb), have failed due to low response rates in the clinical trials (13). Thus, discovering a therapeutic strategy and reducing the risk of disease progression for patients with TNBC is very important.

Isochlorogenic acid c (ICAC), a di-O-caffeoyl derivative of chlorogenic acid (ChA), is isolated from the buds of Lonicera japonica and Cynara scolymus L. (globe artichoke) $(14,15)$. Previous reports have demonstrated that artichoke extracts (AEs) exhibited antitumor effects, such as triggering apoptosis and decreasing the motility and invasive activity on human breast cancer cell line, MDA-MB-231 (isolated from pleural effusions of a breast cancer patient) (16). However, the exact anti-tumor components of AEs and the mechanisms involved in reducing invasiveness are largely unknown. The potential effects of ChA and its derivatives, the most representative components of AEs, against MDAMB-231 cells is not fully elucidated.

In order to find novel compounds from the derivatives of ChA to promote the goal of the development of drugs against TNBC, we previously screened several components from the derivatives of $\mathrm{ChA}$. Using this approach, we successfully identified that ICAC could change cell morphology, migration and invasion of MDA-MB-231 cells in a dosedependent manner. We sought to elucidate the mechanisms by which ICAC regulates the expression and activity of EGFR signaling pathway, thereby contributing to TNBC metastasis. We found that the levels of phosphorylation of EGFR and extracellular signal regulated kinase1/2 (ERK1/2), a downstream signaling molecule of EGFR, were greatly suppressed by treatment with ICAC in MDA-MB-231 cells.
In addition, we observed that treatment of MDA-MB-231 cells with ICAC significantly inhibited EMT, which was rescued by EGF administration. Further biochemical assays confirmed that ICAC down-regulates EGFR phosphorylation in MDA-MB-231 cells along with cellular EMT through EGFR/phospholipase $\mathrm{C} \gamma \quad(\mathrm{PLC} \gamma) / \mathrm{ERK}^{1} \frac{1}{2} / \mathrm{Slug}$ signaling pathway. Therefore, we suggest that ICAC blocks the EGFR signaling pathway and may be used as an effective drug for the inhibition of metastasis of TNBC.

\section{Materials and Methods}

Chemicals and antibodies. ICAC and its derivatives were purchased from ChemFaces (Wuhan, P.R China) and dissolved in methanol to produce a stock solution. 3-(4, 5-Dimethyl-2-thiazolyl)-2, 5-diphenyl2H-tetrazolium bromide (MTT), propidium iodide (PI) and Giemsa stain solution were purchased from Sigma-Aldrich (St. Louis, MO, USA). Antibodies against EGFR, phospho-Tyrosine (P-Tyr-1000), phospho- PLC $\gamma$ (pPLC $\gamma$ ), PLC $\gamma$, phospho-MAPK family antibody sampler kit/ MAPK family antibody sampler kit, slug, vimentin, Ecadherin, and related secondary antibodies were purchased from Cell Signaling Technology (Beverly, CA, USA). $\beta$-actin antibody and radio immunoprecipitation assay (RIPA) lysis buffer were purchased from Santa Cruz Biotechnology (Dallas, TX, USA).

Cell culture. Cancer cells from human breast, namely, MDA-MB231, Hs578T, MCF-7 and SKBR3 cells were included in this study. The cells were obtained from the Bioresources Collection and Research Center, Food Industry Research and Development Institute (BCRC, Hsinchu, Taiwan). These cells were maintained in medium (Hyclone Laboratories, Logan, UT, USA) specific to each cell line with containing $10 \%$ fetal bovine serum (Gibco, Carlsbad, CA, USA), 100 units $/ \mathrm{ml}$ penicillin, $100 \mu \mathrm{g} / \mathrm{ml}$ streptomycin (Invitrogen, Carlsbad, CA, USA), and $100 \mu \mathrm{M}$ non-essential amino acids (Gibco, Carlsbad, CA, USA) in a humidified atmosphere containing $5 \% \mathrm{CO}_{2}$ at $37^{\circ} \mathrm{C}$.

RNA isolation and RT-PCR analysis. Total RNA was isolated from cells using Trizol reagent as described by the manufacturer (Thermo, West Palm Beach, FL, USA). RT-PCR assay was performed according to Chang et al. (17). The sequences of specific primers were as follows: MMP-2 sense, 5'-TCTCCTGACATTGAC CTTGGC-3', and MMP-2 antisense: 5'-CAAGGTGCTGGCTGAG TAGATC-3'; MMP-9 sense, 5'-TTGACAGCGACAAGA AGTGG3', and MMP-9 antisense, 5'-CCCTCAGTGAAGCGGTA CAT-3'; GAPDH sense, 5'-GGCCTCCAAGGAGTAAGACC-3', and GAPDH antisense: 5'-AGGGGTCTACATGGCAACTG-3'. The RTPCR products were identified by $2 \%$ agarose gel electrophoresis.

Cell viability, migration and invasion assays. Cell cytotoxicity and viability were determined by measuring the activity of mitochondrial malate dehydrogenase $(\mathrm{mMDH})$ with MTT assay (18). Basically, cells were seeded in 24-well plates at $1 \times 10^{4}$ cells/well and cultured in DMEM containing $10 \% \mathrm{FBS}$ at $37^{\circ} \mathrm{C}$. Cell proliferation was determined at $48 \mathrm{~h}$ after seeding by adding MTT and incubating the cells further for $3 \mathrm{~h}$.

Migration assay was performed using the ibidi Culture-Insert (ibidi GmbH, Munich, Germany) and Millicell 24-well hanging inserts with $8 \mathrm{~mm}$ PET membranes (EMD Millipore, Billerica, MA, 
USA). The ibidi Culture-Insert was mainly developed to replace classical scratch assays. Silicon inserts, placed on a cell culture surface, provide two cell culture reservoirs. These two chambers are separated with a $500 \mu \mathrm{m}$ thick wall. For Millicell hanging cell culture inserts, the lower compartment was filled with DMEM containing $20 \%$ FBS. Cells with ICAC treatment were placed in the upper part of the chamber and incubated for $16 \mathrm{~h}$. After incubation, the cells were fixed with methanol and stained with crystal violet for $1 \mathrm{~h}$. The cells on the upper surface of the filter were removed with a cotton swab. The filters were then rinsed in distilled water until no additional stain leaching was observed. The cells were then air-dried for $15 \mathrm{~min}$. The migratory phenotypes were determined by counting the cells that migrated to the lower side of the filter through microscopy at $100 \times$ magnification. For each membrane, a total of 4 fields were selected at random and counted, and each sample was assayed in triplicate.

The invasion assay was performed using Millicell 24-well hanging inserts. The upper side was pre-coated with $10 \mu \mathrm{g} / \mathrm{ml}$ Matrigel (BD Biosciences, Bedford, MA, USA). Cells with ICAC treatment were placed in the upper part of the chamber and incubated at $37^{\circ} \mathrm{C}$ for $24 \mathrm{~h}$. The experimental procedures were identical to the migration assay procedures.

Cell cycle and apoptosis analysis. After treatment, cells were washed twice with phosphate-buffered saline (PBS; Invitrogen, Carlsbad, CA, USA) and then fixed in $70 \%$ ethanol overnight at $20^{\circ} \mathrm{C}$. Fixed cells were stained with a solution containing $10 \mu \mathrm{g} / \mathrm{ml}$ of propidium iodide (PI; Sigma, St. Louis, MO, USA), $100 \mu \mathrm{g} / \mathrm{ml}$ of RNase A (Sigma, St. Louis, MO, USA) and 0.1\% Triton X-100 (Sigma, St. Louis, MO, USA) at $37^{\circ} \mathrm{C}$ in the dark. After 30 min, cell cycle distribution was performed using FACScan (BD Biosciences, Bedford, MA, USA) flow cytometry. For apoptosis assay, MDAMB-231 cells with ICAC treatment were assessed using the Annexin V-FITC/7-AAD kit (Beckman Coulter, CA, USA). The kit is an apoptosis detection product based on the binding properties of Annexin V to phosphatidylserine (PS) and on the specificity of 7 amino-actinomycin D (7-AAD) for DNA guanine-cytosine base pair.

Co-immunoprecipitation and immunoblotting analysis. For coimmunoprecipitation, cells were lysed with cold lysis buffer $(142.5 \mathrm{mM}$ $\mathrm{KCl}, 5 \mathrm{mM} \mathrm{MgCl}{ }_{2}, 10 \mathrm{mM}$ HEPES [pH7.2], $0.2 \%$ Nonidet P-40 (NP-40), and $1 \mathrm{mM}$ EGTA) containing $2 \mathrm{mM}$ phenylmethylsulfonyl fluoride (PMSF), $2 \mu \mathrm{g} / \mathrm{ml}$ aprotinin, $2 \mu \mathrm{g} / \mathrm{ml}$ leupeptin, $1 \mathrm{mM} \mathrm{NaF}$, and $1 \mathrm{mM} \mathrm{Na} \mathrm{VO}_{4}$. Lysates were then centrifuged at $16,000 \times \mathrm{g}$ for 10 minutes at $4^{\circ} \mathrm{C}$. Equal amounts of protein extracts were subjected to immunoprecipitation and immunoblotting analysis following methods described previously (19). Approximately $500 \mu \mathrm{g}$ of lysates were subjected to immunoprecipitation with specific antibodies. After incubating overnight at $4^{\circ} \mathrm{C}$, the specific proteins or peptides were precipitated with protein $\mathrm{A} / \mathrm{G}$ sepharose beads (Thermo, West Palm Beach, FL, USA) and purified using according to the manufacturer's protocol.

For Western blotting, cells were lysed in RIPA buffer containing protease and phosphatase inhibitors (Roche, Mannheim, Germany). Equal amounts of protein extracts were subjected to immunoblot following methods described previously (19).

Statistical analysis. The data are expressed as mean \pm standard errors (SE) of three independent experiments and were analyzed by ANOVA. Pearson's chi-squared test and Student's $t$-test were used in two-group comparisons. $p<0.05$ was considered as statistically significant.

\section{Results}

Basal EGFR expression level is high in TNBC cells. Activation of EGFR-induced signaling pathway has been correlated with cancer metastasis in various tumors (20). To evaluate whether EGFR status is associated with the mortality of TNBC, we compared the OS and different EGFR expression levels in patients with TNBC. The expression data of 355 TNBC patients including 117 EGFR-high and 138 EGFR-low patients from Kaplan-Meier plotter microarray database (http:// kmplot.com/analysis/index.php?p=service\&cancer=breast) was downloaded and performed with univariate analysis (KaplanMeier method). As shown in Figure 1A, we found that clinical EGFR expression had significant associations with the OS $(p<0.05)$.

Immunoblot analysis showed that EGFR level was significantly higher in TNBC (MDA-MB-231 and Hs578T) cells than in non-TNBC (MCF-7 and BT-474) cells (Figure $1 \mathrm{~B})$. EMT is one of the major mechanisms involved in breast cancer metastasis. Next, the protein levels of EMT-related markers, including slug, vimentin, and E-cadherin were examined. As shown in Figure 1B, the levels of slug and vimentin protein expression increased significantly in TNBC cells compared with that in non-TNBC cells. In comparison with MCF-7 and BT-474 cells, decreased protein level of Ecadherin was observed in the MDA-MB-231 and Hs578T cells. These findings suggest that a high EGFR expression is associated with the enhanced invasiveness and metastasis of BC cells. Moreover, EGFR is considered a potential therapeutic target and negative prognostic biomarker in TNBC (21). In addition, there is no effective therapy for estrogen-independent breast cancer. We sought to focus on MDA-MB-231 cells, expressing the highest level of EGFR protein, which provided the interpretation for testing ChA and its derivatives against highly invasive breast cancer phenotypes.

ICAC alters morphology but cannot affect growth, apoptosis, and cell cycle distribution of ICAC-treated MDA-MB-231 cells. To find a particular compound with a novel antitumor mechanism against TNBC, we screened several compounds of ChA and its derivatives. As shown in Figure 2A, vehicletreated MDA-MB-231 cells displayed a fibroblast-like morphology. However, they showed a cobblestone/epitheliallike morphology after treatment with ICAC for $48 \mathrm{~h}$. Proliferation is important for tumor invasion. To determine the effect of ICAC treatment on the viability of MDA-MB231 cells, MTT assay was performed. ICAC-treated MDAMB-231 cells did not influence cell viability within 24 and $48 \mathrm{~h}$ (Figure 2B). To determine whether the apoptotic effect could be observed in ICAC-treated MDA-MB-231 cells, apoptotic cell death was assessed by flow cytometry assay after double staining with Annexin V and 7-AAD. Our data 


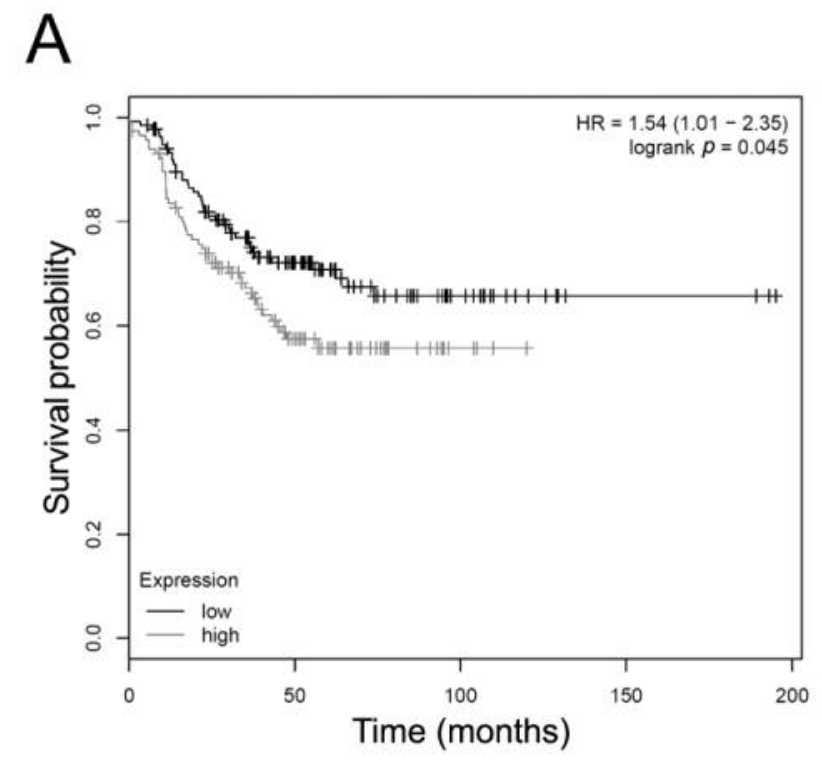

B

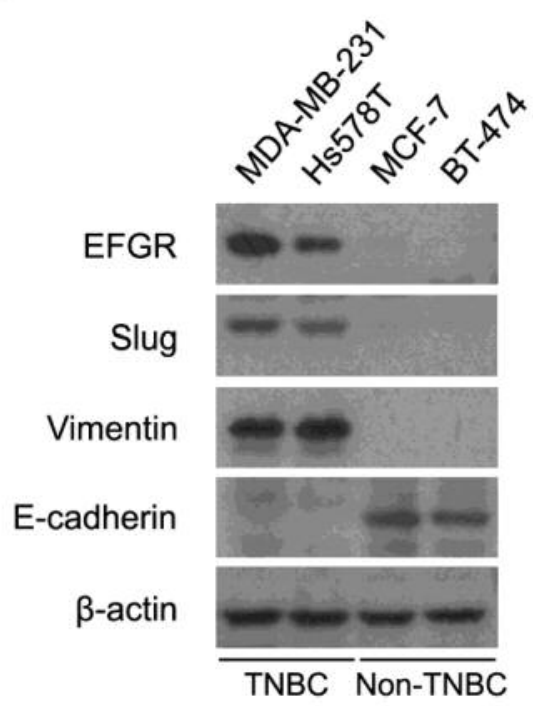

Figure 1. Basal EGFR, slug, and vimentin expression levels were increased in TNBC cells. (A) Survival curve for different EGFR expression levels in patients with TNBC were analyzed using Kaplan-Meier plotter microarray database. (B) Immunoblotting analyses and correlations of EGFR and EMT markers expression in human breast cancer. The results are representative of three independent experiments.

showed that ICAC did not cause a significant change in apoptotic index in MDA-MB-231 cells compared with the control (Figure 2C). In order to evaluate whether ICAC affected cell cycle distribution of ICAC-treated MDA-MB231 cells, we performed FACS analysis at $48 \mathrm{~h}$ after ICAC treatment. Cell cycle analysis showed that ICAC had no effect on alteration of cell cycle progression in MDA-MB231 cells (Figure 2D). Based on these results, ICAC have no effect on growth, apoptosis, and cell cycle distribution of MDA-MB-231 cells.

ICAC induces mesenchymal-epithelial transition (MET) in $M D A-M B-231$ cells. The distinct morphology evident in MDA-MB-231 cells treated with ICAC was reminiscent of cells that have undergone MET (Figure 2A). To investigate this possibility, we examined the expression of mesenchymal and epithelial markers associated with MET (22). Cells treated with ICAC displayed a decreased expression of mesenchymal markers, including slug and vimentin, as well as an increased expression of the epithelial marker, Ecadherin, in a dose-dependent manner whereas control cells resembled parental cells (Figure 3A). When MET is in progress, the mesenchymal cells tend to have an epithelial morphology, hence the expression of mesenchymal markers decreases and expression of epithelial markers increases. Both the morphological and the molecular changes suggested that MDA-MB-231cells might undergo reversal of EMT with exposure to ICAC than in control cells. Cell migration and invasion are indispensable in tumor metastasis, so both migration and invasion assay were performed to evaluate the role of ICAC in this process. As shown in Figure 3B, the result of the wound healing assay showed that ICAC retarded significantly MDA-MB-231 cell migration. Next, the invasive potential of MDA-MB-231 cells in the Matrigelcoated Boyden chamber was analyzed in the absence or presence of ICAC. The number of MDA-MB-231 cells exposed to ICAC that moved through the membrane of the Matrigel chamber was about $76 \%$ less than that of control cells (Figure 3D). In addition, ICAC reduced the migration ability of MDA-MB-231 cells as proven by a $71 \%$ decrease in cell movement (Figure 3C). These findings indicate that ICAC reduces the invasive capacity and migration ability of MDA-MB-231 cells.

Down-regulation of EGFR phosphorylation by ICAC. Expression and phosphorylation of EGFR in tumor samples have been reported to correlate with tumor stage, progression, and metastasis (23). In order to clarify the involvement of ICAC in EGFR signaling pathway in MDAMB-231 cells, cells were treated with the indicated concentration of ICAC for $48 \mathrm{~h}$. Here, we would analyze several major components of signaling pathways downstream of the EGFR including MAPK, PLC, and PI3K which have demonstrated as having potential effects in EMT (24-26). As shown in Figure 4A, the phosphorylation of EGFR and its downstream signaling molecule PLC $\gamma$ and 
A

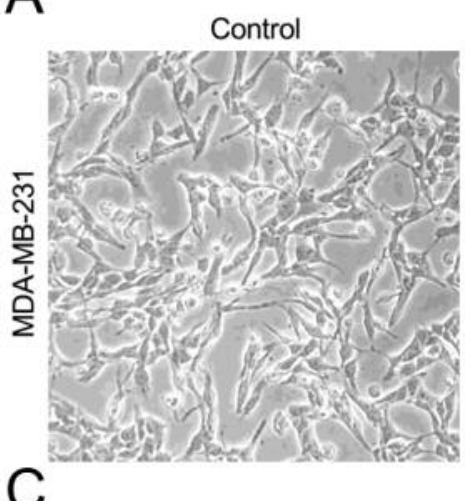

Control
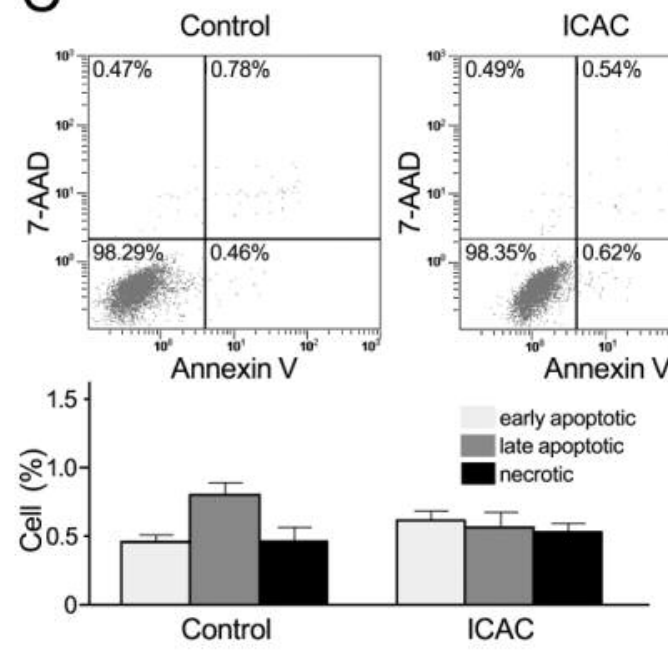

ICAC

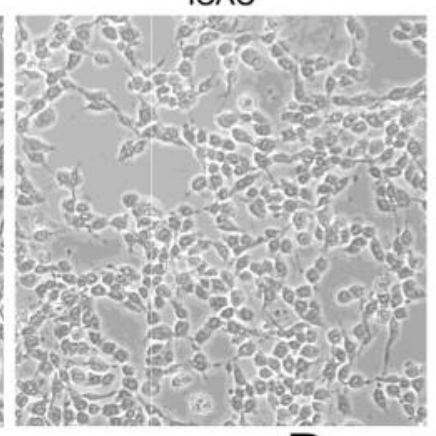

B
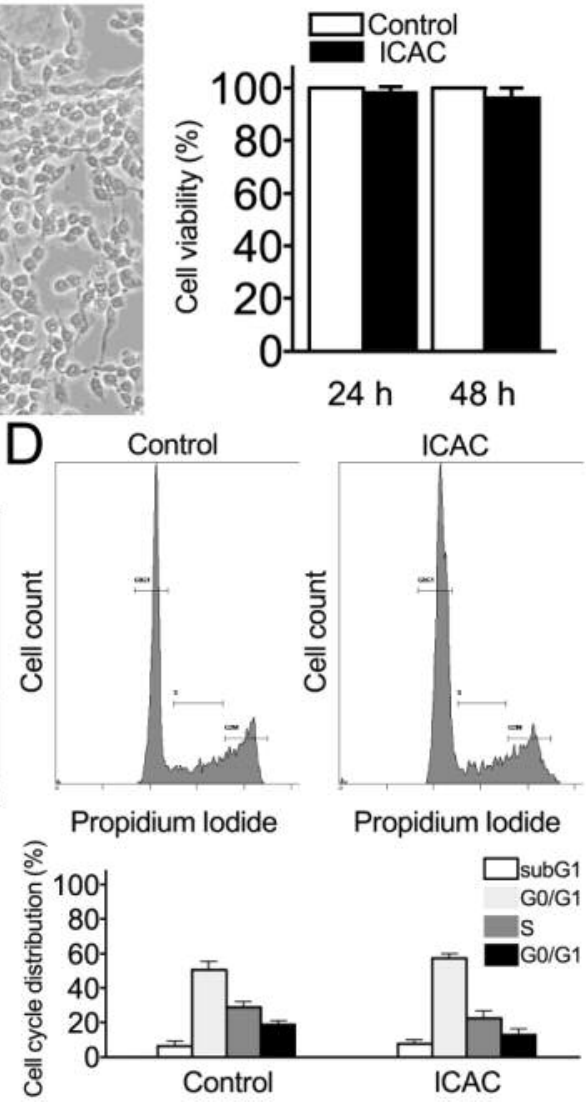

Figure 2. Morphological changes of ICAC-treated MDA-MB-231 cells. (A) Images captured using phase-contrast microscopy revealed changes in morphology from spindle shaped (control) to cobblestonelepithelial-like (ICAC) in MDA-MB-231 cells with ICAC treatment. (B) The effect of treatment with vehicle (control) or ICAC on cell proliferation for 24 and $48 \mathrm{~h}$ was determined by the MTT assay. Absorbance values obtained from MDA-MB-231 cells treated with vehicle were taken as 100\%. (C) Analysis of apoptosis in ICAC-treated MDA-MB-231 cells by FACS. The viable cells are Annexin $V-7 A A D-(Q 3)$. The early apoptotic cells are Annexin $V^{+} 7 A A D^{-}(Q 4)$. The late apoptotic cells are Annexin $V+7 A A D+(Q 2)$. (D) Flow cytometry of ICAC-treated MDA-MB-231 cells assessed for their distribution in the cell cycle. Values are means $\pm S E$ of the percentage of cells in the subG1 (white bars), $G_{0} / G_{1}$ (light gray bars), $S$ (dark gray bars) and $G 2 / M$ (black bars) phases of the cell cycle. Data are representative of three independent experiments.

ERK1/2 were down-regulated with ICAC treatment. By contrast, the ICAC-treated proteins expression including PI3K signaling pathway were not altered (data not shown).

ICAC attenuates the expression of MMP-9 in MDA-MB231cells. Previous studies have shown that matrix metalloproteinases (MMP)-2 and -9 play an important role in breast cancer metastasis through the degradation of the extracellular matrix (ECM) (27). To examine whether ICAC regulates invasion by altering the expression of MMP-2 and MMP-9, RT-PCR analysis demonstrated that the mRNA levels of MMP-9 were inhibited in MDA-MB-231 cells in response to ICAC treatment (Figure 4A). At $50 \mu \mathrm{M}$, ICAC significantly down-regulated MMP-9 expression. In contrast, there was no difference in $M M P-2$ mRNA levels (data not shown). These results indicate that ICAC could suppress the transcription of MMP-9 gene in MDA-MB-231 cells.

EGF rescues the down-regulating EGFR phosphorylation signaling pathway by ICAC. A previous study reported that EGF stimulates a strong migratory response in MDA-MB231 cells (28). To verify the effect of ICAC on EGF/EGFR signaling pathway in MDA-MB-231 cells pretreated with ICAC, were incubated with EGF . Our data showed that the phosphorylation of EGFR and downstream signaling proteins including PLC $\gamma$ and ERK1/2 were increased by EGF treatment (Figure 4B). We also found that treatment with EGF could sufficiently rescue not only the mesenchymal 


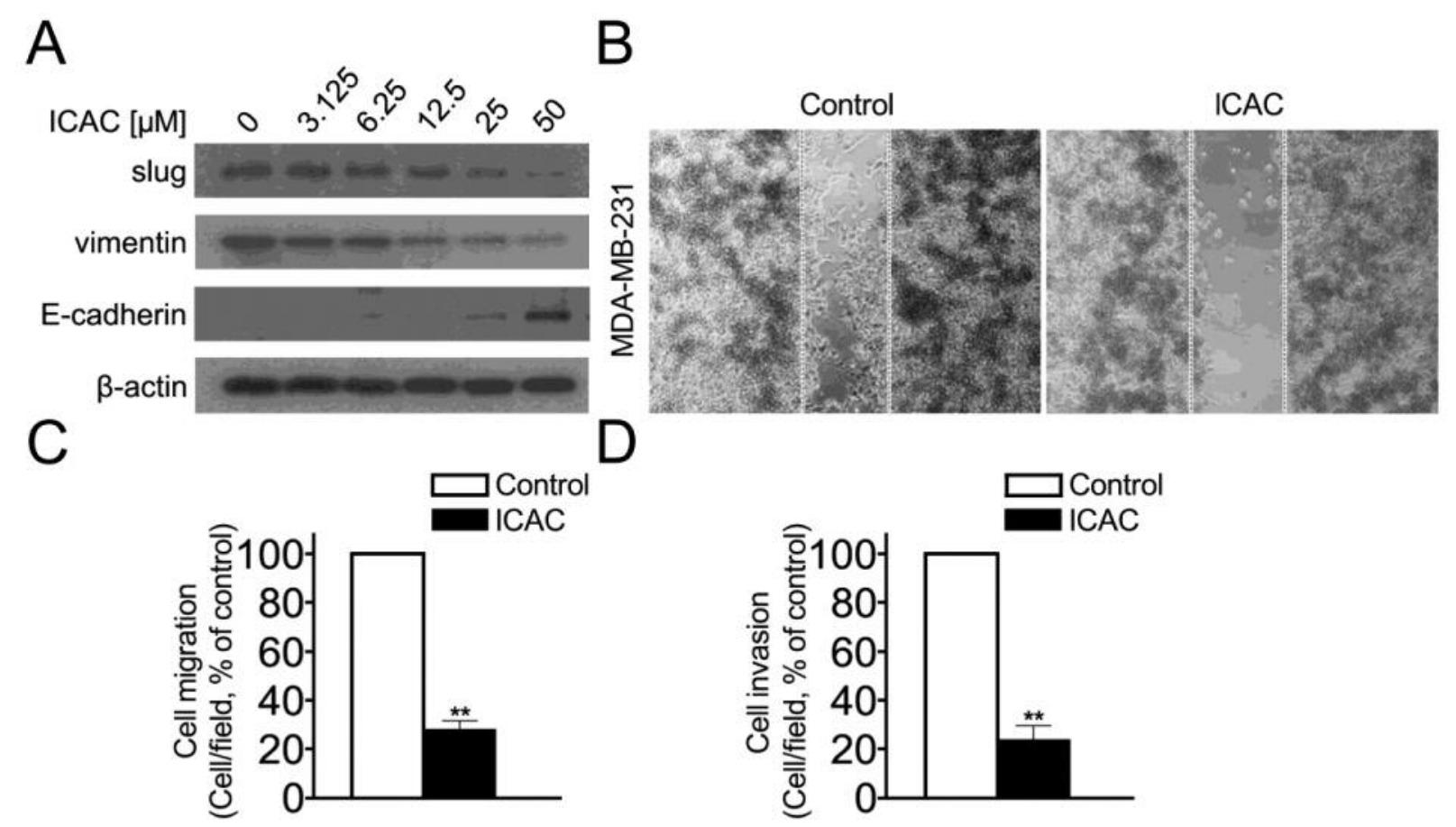

Figure 3. ICAC induces mesenchymal-epithelial transition (MET) in MDA-MB-231cells. (A) Immunoblotting analysis of slug, vimentin, and E-cadherin expression in ICAC-treated MD-MB-231 cells. Cells were treated with vehicle or increasing concentrations of ICAC (3.125 to $50 \mu M$ ) for $48 \mathrm{~h}$, then whole-cell extracts were subjected to western blot analysis for slug, vimentin, E-cadherin, and $\beta$-actin (loading control) proteins, respectively. (B) Confluent culture of MDA-MB-231 cells were wounded by using the ibidi Culture-Insert and the cells were treated without and with indicated concentrations of ICAC. The wound was photographed with an inverted microscope. In vitro migration $(C)$ and invasion $(D)$ capacities of the MDA-MB-231 cell line upon treatment with ICAC. MDA-MB-231 cells were treated with $50 \mu M$ ICAC. After treatment, these cells were allowed to migrate through uncoated transwells for $16 \mathrm{~h}(C)$ or invade through Matrigel-coated transwells for $24 \mathrm{~h}(D)$. The number of cells at the bottom of the transwell filters was counted at the end of each assay. Results are presented as means \pm SE from three independent experiments, performed in duplicate, all versus control (cells treated with methanol, vehicle).

markers, including slug and vimentin, but also the epithelial marker, E-cadherin. These results show that ICAC exerts its anti-EMT effect by altering EGFR signaling pathway in MDA-MB-231 cells. EGF has also been shown to induce MMP-9 expression through ERK1/2-dependent pathway in breast cancer cells (29). Therefore, we assessed whether EGF could influence the effect of ICAC-reduced MMP-9 expression. ICAC-reduced MMP-9 expression was rescued by EGF (Figure 4B). Altogether, our results suggested that ICAC modulates the EMT process through the inhibition of the EGFR signaling pathway in MDA-MB-231 cells.

\section{Discussion}

ICAC, one of the phenolic phytochemicals, extracted from plants including artichoke and Lonicera japonica. Although ICAC has been implicated in antiviral potency, its underlying molecular mechanisms in tumorigenesis remain unclear and should be investigated (30). Recent studies have shown that natural phytochemicals containing phenolic compounds have antitumor and anti-metastatic properties (16). A number of studies have shown certain plant extracts, rather than a single compound, had significant cancer chemopreventive properties. In contrast to those studies, the purpose of this study was to improve our understanding over the biological role and molecular mechanisms of single phytochemical with potential antitumor effect on TNBC cells. Because of the absence of a suitable target $\left(\mathrm{ER}^{-}\right)$, there is no effective therapy for TNBC patients. Developing a new therapeutic manner for TNBC is absolutely necessary.

In the present study, we have found the molecular mechanisms supporting the antitumor activities of ICAC from the derivative of ChA on a human TNBC cell line. We provided an assay for testing ICAC as a chemopreventive agent against invasive and hormone resistant breast cancer phenotypes. Finally, we demonstrated that ICAC attenuated the migratory capability, in which, EGFR-mediated downregulation of PLC $\gamma$, ERK1/2, and MMPs signal pathways were involved, and then having an anti-metastatic effect by attenuating the mesenchymal traits. 


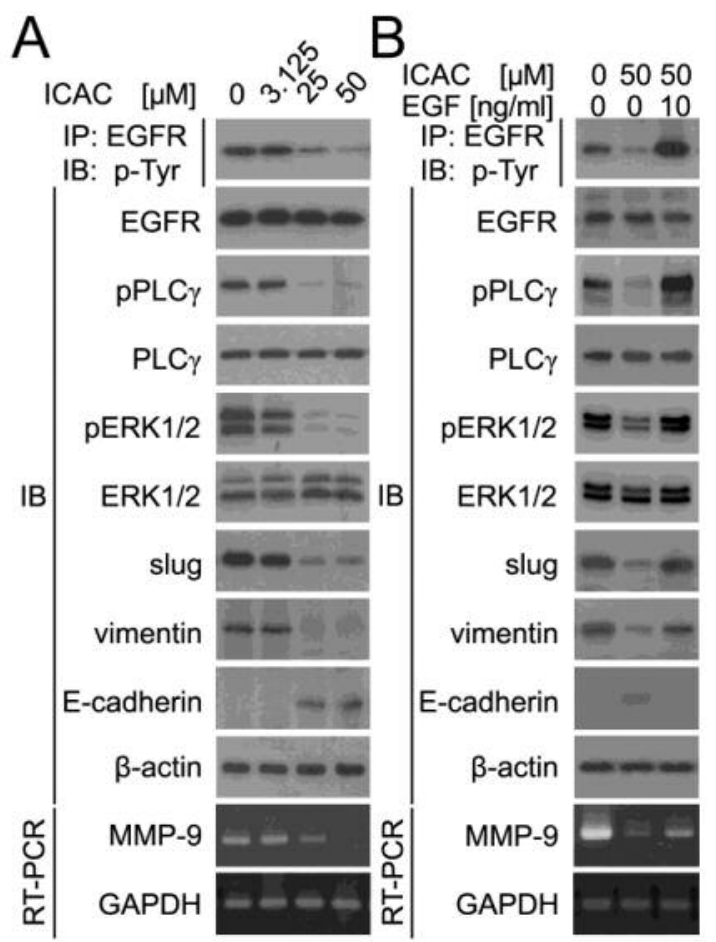

Figure 4. ICAC down-regulated the phosphorylation of EGFR that is rescued by EGF in MDA-MB-231 cells. (A) Cells were treated with vehicle or ICAC for $48 h$, and then the protein expression was measured by Western blot analysis. (B) Cells were pretreated with ICAC for $24 \mathrm{~h}$, and then they were co-treated with EGF for $24 \mathrm{~h}$. The levels of each protein expression were analyzed by western blotting. The results are representative of three independent experiments.

The cytotoxicity of ICAC was evaluated in the MDA-MB231 cell model. MDA-MB-231 cells, which are highly aggressive TNBC cells, were chosen as a cellular model to assess the antitumor effects of ICAC. ICAC-treatment resulted in increased expression of epithelial markers and decreased expression of mesenchymal markers in MDA-MB231 cells. As these cells normally show increased expression of mesenchymal markers in culture, it seems that ICACtreatment causes the mesenchymal to epithelial switch by reduction of mesenchymal markers and induction of epithelial markers. The results indicated that ICAC effectively reduces the migration and invasion of TNBC cells, but could not have an effect on non-TNBC cells (data not shown). Experimentally-induced EMT was reduced following exposure of MDA-MB-231 cells to ICAC or EGF as evidenced by modulation in the expression levels of EMTmarkers. Treatment with EGF could rescue the EMT phenotype, which allows tumor cells to obtain the ability to migrate and to invade adjacent tissues. Tumor metastasis, including cell adhesion, proteolytic degradation of extracellular matrix (ECM) and cell migration, is critically associated with the acquisition of an EMT phenotype (31). Therapy for metastasis is based on the blocking of one of these pathways. In addition, there is evidebce that cell surface receptors mediate tumor metastasis . Cell surface receptors are mostly overexpressed and up-regulated in transformed cells. EGFR is frequently up-regulated in breast cancer or metastatic breast cancers, especially in TNBC (32). Furthermore, EGFR is constantly overexpressed in metastatic breast cancers (33). Activation of EGFR is associated with a poor prognosis in several tumor types, including TNBC (3436). EGFR/p53 IHC phenotype appears to be associated with prognosis in patients with operable TNBC receiving conventional adjuvant chemotherapy (5). Therefore, EGFR could be a therapeutic target for the treatment of TNBC. EGFR is a transmembrane tyrosine kinase receptor member that belongs to the HER/ErbB family. Phosphorylation of the intracellular domain of EGFR activates downstream signaling pathways including PLC $\gamma$, Ras, MAPK and PI3K/Akt that lead to transcriptional regulation of genes involved in cell proliferation, survival and drug resistance $(36,37)$. PLC $\gamma$ would be activated by EGFR tyrosine kinase through its $\mathrm{SH} 2$ domains $(38,39)$. It has been indicated that PLC $\gamma$ implicates cell motility through modulating cytoskeletal dynamics. PLC $\gamma$ could associate with actin filaments through its $\mathrm{SH} 3$ domain, and then modulate actin assembly, functionally important steps in the regulation of metastasis $(38,40)$.

Metastasis is a complex progression involving several stages, including invasiveness, intravasation, extravasation, and growth in other organs. Tumor invasion across the ECM is thought to be one of the critical stages. The ability of tumor cells to degrade ECM components is associated with the presence of cells with metastatic potential (41). Several proteolytic enzymes, such as MMP-2 and MMP-9 (gelatinase $\mathrm{B}$, type IV collagenase), are thought to be involved directly in the migration, invasion and metastasis of tumor cells, and associated with the prognosis factor $(42,43)$. ERK $1 / 2$ has been implicated in the modulation of EMT by phosphorylation and activation of EMT participators in the breast cancer cell $(44,45)$. ERK1/2 is involved in the modulation of MMP-9 level in MDA-MB-231 cells (46). In this study, endogenous MMP-9 mRNA expression was markedly down-regulated in cells with exposure to ICAC. This finding suggests that the down-regulation of MMP-9 is involved in the ICAC-mediated reduction in cell migration and invasion potential.

We found that ICAC-treatment significantly inhibited EGFR phosphorylation and reduced the phosphorylation of PLC $\gamma$, ERK1/2 and MMP-9 mRNA expression in MDAMB-231 cells. On the other hand, we did not observe any effect on PI3K and Akt phosphorylation. These results might indicate that PI3K/Akt signaling is not involved in ICACmediated anti-metastatic effects. 
Overall, our in vitro findings suggested that ICAC restrained breast cancer cell migration and invasion, at least in part, by down-regulation of EGFR/PLC $\gamma / \mathrm{ERK}^{1 / 2} / 2 / \mathrm{slug}$ signaling and the reversal of EMT. In addition, ICAC also suppressed the expression of MMP-9, which is a significant enzyme for the degradation of ECM proteins such as type I and IV collagen during invasion. Therefore, ICAC could be further developed as an effective drug candidate against tumor invasion and metastasis of human TNBC through suppression of EGFR signaling.

\section{Acknowledgements}

This study was supported by the Research Fund of Tung's Taichung MetroHarbor Hospital (TTMHH-106R0024) and Chang Bing Show Chwan Memorial Hospital (RD106004).

\section{References}

1 Foulkes WD, Smith IE and Reis-Filho JS: Triple-negative breast cancer. N Engl J Med 363: 1938-1948, 2010.

2 Dent R, Trudeau M, Pritchard KI, Hanna WM, Kahn HK, Sawka CA, Lickley LA, Rawlinson E, Sun P and Narod SA: Triplenegative breast cancer: clinical features and patternsof recurrence. Clin Cancer Res 13: 4429-4434, 2007.

3 Carey L, Winer E, Viale G, Cameron D and Gianni L: Triplenegative breast cancer: disease entity or title of convenience? Nat Rev Clin Oncol 7: 683-692, 2010.

4 Normanno N, De Luca A, Bianco C, Strizzi L, Mancino M, Maiello MR, Carotenuto A, De Feo G, Caponigro F and Salomon DS: Epidermal growth factor receptor (EGFR) signaling in cancer. Gene 366: 2-16, 2006.

5 Levva S, Kotoula V, Kostopoulos I, Manousou K, Papadimitriou C, Papadopoulou K, Lakis S, Koukoulias K, Karavasilis V, Pentheroudakis G, Balassi E, Zagouri F, Kaklamanos IG, Pectasides D, Razis E, Aravantinos G, Papakostas P, Bafaloukos D, Rallis G, Gogas H and Fountzilas G: Prognostic evaluation of epidermal growth factor receptor (EGFR) genotype and phenotype parameters in triple-negative breast cancers. Cancer Genomics Proteomics 14: 181-195, 2017.

6 Li MH, Tsai JT, Ting LL, Lin JC and Liu YC: Comparison of thoracic radiotherapy efficacy between patients with and without EGFR-mutated lung adenocarcinoma. In Vivo 32: 203 209, 2018.

7 Borghetti P, Bonù ML, Roca E, Pedretti S, Salah E, Baiguini A, Greco D, Triggiani L, Maddalo M, Levra NG, Alongi F, Magrini SM and Buglione M: Radiotherapy and tyrosine kinase inhibitors in stage IV non-small cell lung cancer: Real-life experience. In Vivo 32: 159-164, 2018.

8 Sluga R, VAN DEN Borne BEEM, Roepman P, Peters BJM, Kastelijn EA and Schramel FMNH: Utilization of molecular testing and survival outcomes of treatment with first- or second-line tyrosine kinase inhibitors in advanced non-small cell lung cancer in a dutch population. Anticancer Res 38 : 393-400, 2018.

9 Wise R and Zolkiewska A: Metalloprotease-dependent activation of EGFR modulates $\mathrm{CD} 44^{+} / \mathrm{CD} 24^{-}$populations in triplenegative breast cancer cells through the MEK/ERK pathway. Breast Cancer Res Treat 166: 421-433, 2017.
10 Tan DS, Marchio C, Jones RL, Savage K, Smith IE, Dowsett M and Reis-Filho JS: Triple negative breast cancer: molecular profiling and prognostic impact in adjuvant anthracycline-treated patients. Breast Cancer Res Treat 111: 27-44, 2008.

11 Eralp Y, Derin D, Ozluk Y, Yavuz E, Guney N, Saip P, Muslumanoglu M, Igci A, Kücücük S, Dincer M, Aydiner A and Topuz E: MAPK overexpression is associated with anthracycline resistance and increased risk for recurrence in patients with triple-negative breast cancer. Ann Oncol 19: 669674, 2008.

12 Sun XD, Liu XE and Huang DS: Curcumin induces apoptosis of triple-negative breast cancer cells by inhibition of EGFR expression. Mol Med Rep 6: 1267-1270, 2012.

13 Nakai K, Hung MC and Yamaguchi H: A perspective on antiEGFR therapies targeting triple-negative breast cancer. Am J Cancer Res 6: 1609-1623, 2016.

14 Guo W, Wang L, Gao Y, Zhao B, Wang D, Duan W and Yu Z: Isolation of Isochlorogenic acid isomers in flower buds of Lonicera japonica by high-speed counter-current chromatography and preparative high performance liquid chromatography. $\mathrm{J}$ Chromatogr B Analyt Technol Biomed Life Sci 981-982: 27-32, 2015.

15 Colak E, Ustuner MC, Tekin N, Burukoglu D, Degirmenci I and Gunes HV: The hepatocurative effects of Cynara scolymus L. leaf extract on carbon tetrachloride-induced oxidative stress and hepatic injury in rats. Springerplus 5: 216, 2016.

16 Mileo AM, Di Venere D, Linsalata V, Fraioli R and Miccadei S: Artichoke polyphenols induce apoptosis and decrease the invasive potential of the human breast cancer cell line MDAMB231. J Cell Physiol 227: 3301-3309, 2012.

17 Chang IC, Chiang TI, Lo C, Lai YH, Yue CH, Liu JY, Hsu LS and Lee CJ: Anemone altaica induces apoptosis in human osteosarcoma cells. Am J Chin Med 43: 1031-1042, 2015.

18 Lee CJ, Yue CH, Lin YY, Wu JC and Liu JY: Antitumor activity of acriflavine in human hepatocellular carcinoma cells. Anticancer Res 34: 3549-3556, 2014.

19 Lee CJ, Hsu LS, Yue CH, Lin H, Chiu YW, Lin YY, Huang CY, Hung MC and Liu JY: MZF-1/Elk-1 interaction domain as therapeutic target for protein kinase $\mathrm{C} \alpha$-based triple-negative breast cancer cells. Oncotarget 7: 59845-59859, 2016.

20 Hsieh CY, Tsai PC, Tseng CH, Chen YL, Chang LS and Lin SR: Inhibition of EGF/EGFR activation with naphtho[1,2-b]furan4,5-dione blocks migration and invasion of MDA-MB-231 cells. Toxicol In Vitro 27: 1-10, 2013.

21 Uray IP and Brown PH: Chemoprevention of hormone receptornegative breast cancer: New approaches needed. Recent Results Cancer Res 188: 147-162, 2011.

22 Hajra KM, Chen DY and Fearon ER: The SLUG zinc-finger protein represses E-cadherin in breast cancer. Cancer Res 62: 1613-1618, 2002.

23 Wells A: Tumor invasion: role of growth factor-induced cell motility. Adv Cancer Res 78: 31-101, 2000.

24 Klemke RL, Cai S, Giannini AL, Gallagher PJ, de Lanerolle P and Cheresh DA: Regulation of cell motility by mitogenactivated protein kinase. J Cell Biol 137: 481-492, 1997.

25 Wennstrom S, Hawkins P, Cooke F, Hara K, Yonezawa K, Kasuga M, Jackson T, Claesson-Welsh L and Stephens L: Activation of phosphoinositide 3-kinase is required for PDGF-stimulated membrane ruffling. Curr Biol 4: 385-393, 1994. 
26 Chen P, Xie H, Sekar MC, Gupta K and Wells A: Epidermal growth factor receptor-mediated cell motility: phospholipase $\mathrm{C}$ activity is required, but mitogen- activated protein kinase activity is not sufficient for induced cell movement. J Cell Biol 127: 847-857, 1994.

27 Al Dhaheri Y, Attoub S, Arafat K, Abuqamar S, Viallet J, Saleh A, Al Agha H, Eid A and Iratni R: Anti-metastatic and antitumor growth effects of Origanum majorana on highly metastatic human breast cancer cells: inhibition of NFKB signaling and reduction of nitric oxide production. PLoS One 8: e68808, 2013.

28 Price JT, Tiganis T, Agarwal A, Djakiew D and Thompson EW: Epidermal growth factor promotes MDA-MB-231 breast cancer cell migration through a phosphatidylinositol 3'-kinase and phospholipase C-dependent mechanism. Cancer Res 59: 5475$5478,1999$.

29 Kim S, Choi JH, Lim HI, Lee SK, Kim WW, Cho S, Kim JS, Kim JH, Choe JH, Nam SJ, Lee JE and Yang JH: EGF-induced MMP-9 expression is mediated by the JAK3/ERK pathway, but not by the JAK3/STAT-3 pathway in a SKBR3 breast cancer cell line. Cell Signal 21: 892-898, 2009.

30 Cao Z, Ding Y, Cao L, Ding G, Wang Z and Xiao W: Isochlorogenic acid $\mathrm{C}$ prevents enterovirus 71 infection via modulating redox homeostasis of glutathione. Sci Rep 7: 16278, 2017

31 Hanahan D and Weinberg RA: Hallmarks of cancer: The next generation. Cell 144: 646-674, 2011.

32 Nielsen TO, Hsu FD, Jensen K, Cheang M, Karaca G, Hu Z, Hernandez-Boussard T, Livasy C, Cowan D, Dressler L, Akslen LA, Ragaz J, Gown AM, Gilks CB, van de Rijn M and Perou $\mathrm{CM}$ : Immunohistochemical and clinical characterization of the basal-like subtype of invasive breast carcinoma. Clin Cancer Res 10: 5367-5374, 2004.

33 Nicolini A, Carpi A and Tarro G: Biomolecular markers of breast cancer. Front Biosci 11: 1818-1843, 2006.

34 Nicholson RI, Gee JM and Harper ME: EGFR and cancer prognosis. Eur J Cancer 37: S9-15, 2001.

35 Rimawi MF, Shetty PB, Weiss HL, Schiff R, Osborne CK, Chamness GC and Elledge RM: Epidermal growth factor receptor expression in breast cancer association with biologic phenotype and clinical outcomes. Cancer 116: 1234-1242, 2010.

36 El Guerrab A, Bamdad M, Kwiatkowski F, Bignon YJ, PenaultLlorca $\mathrm{F}$ and Aubel C: Anti-EGFR monoclonal antibodies and EGFR tyrosine kinase inhibitors as combination therapy for triple-negative breast cancer. Oncotarget 7: 73618-73637, 2016
37 Schlessinger J: Cell signaling by receptor tyrosine kinases. Cell 103: 211-225, 2000.

38 Chen P, Xie H, Sekar MC, Gupta K and Wells A: Epidermal growth factor receptor-mediated cell motility: Phospholipase C activity is required, but mitogen-activated protein kinase activity is not sufficient for induced cell movement. J Cell Biol 127: 847-857, 1994.

39 Meisenhelder J, Suh PG, Rhee SG and Hunter T: Phospholipase $\mathrm{C}$-gamma is a substrate for the PDGF and EGF receptor proteintyrosine kinases in vivo and in vitro. Cell 57: 1109-1122, 1989.

40 Bar-Sagi D, Rotin D, Batzer A, Mandiyan V and Schlessinger J: SH3 domains direct cellular localization of signaling molecules. Cell 74: 83-91, 1993.

41 Cavallaro U and Christofori G: Cell adhesion in tumor invasion and metastasis: loss of the glue is not enough. Biochim Biophys Acta 1552: 39-45, 2001.

42 Chambers AF, Groom AC and MacDonald IC: Dissemination and growth of cancer cells in metastatic sites. Nat Rev Cancer 2: 563-572, 2002.

43 Mignatti $P$ and Rifkin DB: Biology and biochemistry of proteinases in tumor invasion. Physiol Rev 73: 161-195, 1993.

44 Khan S, Shukla S, Sinha S, Lakra AD, Bora HK and Meeran SM: Centchroman suppresses breast cancer metastasis by reversing epithelial-mesenchymal transition via down-regulation of HER2/ERK1/2/MMP-9 signaling. Int J Biochem Cell Biol 58: $1-16,2015$.

$45 \mathrm{Li} \mathrm{NY}$, Weber CE, Wai PY, Cuevas BD, Zhang J, Kuo PC and Mi Z: An MAPK-dependent pathway induces epithelialmesenchymal transition via Twist activation in human breast cancer cell lines. Surgery 154: 404-410, 2013.

46 Gomes LR, Terra LF, Wailemann RA, Labriola L and Sogayar MC: TGF- $\beta 1$ modulates the homeostasis between MMPs and MMP inhibitors through p38 MAPK and ERK1/2 in highly invasive breast cancer cells. BMC Cancer 12: 26, 2012.
Received January 5, 2018

Revised February 15, 2018

Accepted February 16, 2018 